\title{
LA EXPRESIÓN DEL 'INTERÉS' EN LOS AMICUS CURIAE PRESENTADOS POR LA SOCIEDAD CIVIL ANTE LA CORTE PENAL INTERNACIONAL
}

\author{
THE EXPRESSION OF 'INTEREST' IN THE AMICUS CURIAE PRESENTED BY \\ THE CIVIL SOCIETY BEFORE THE INTERNATIONAL CRIMINAL COURT
}

\section{Carmen Montero Ferrer *}

\section{RESUMEN}

En el estadio actual del Derecho Internacional Público, el acceso del individuo y la sociedad civil organizada a los tribunales internacionales, y su participación en los procedimientos es una constante. En especial, a través de la institución del amicus curiae, tradicionalmente definida como el observador neutral que interviene voluntariamente en un litigio, ayudando al órgano jurisdiccional en la resolución del caso. Esta definición ha ido evolucionando en el plano internacional, de modo que en algunas ocasiones se ha admitido la intervención de amici que manifiesten tener interés en el resultado de los procedimientos. No obstante, la expresión del interés no está explícitamente considerada como un criterio para determinar la admisión o el rechazo del amicus curiae en la normativa de los tribunales penales internacionales. En este sentido, el Estatuto de Roma de la Corte Penal Internacional no es una excepción. Por esta razón, el presente trabajo lleva a cabo un análisis normativo y jurisprudencial de los efectos jurídicos derivados de la expresión del interés en los escritos de amicus presentados por la sociedad civil ante la Corte Penal Internacional.

Palabras clave: amicus curiae, sociedad civil, concepto de interés, Corte Penal Internacional.

\footnotetext{
* Carmen Montero Ferrer es investigadora posdoctoral de la Universidad de Santiago de Compostela (España). Becaria de la Xunta de Galicia (España) y miembro del Grupo de Estudios Internacionales de dicha universidad (GRESIN-GI-1138). Actualmente, está realizando una estancia de investigación en la 'Chaire de recherche du Canada sur la justice internationale pénale et les droits fondamentaux' de la Université Laval (Québec-Canadá) 2018-2020. Este trabajo forma parte del proyecto más amplio "Contribuciones de la sociedad civil a la rendición de cuentas por crímenes internacionales. Especial referencia a la Corte Penal Internacional" financiado por la Convocatoria de axudas de apoio á etapa de formación posdoutoral nas universidades do SUG, nos organismos públicos de investigación de Galicia. Modalidad A. Anteriormente, realizó una estancia de investigación en la Comisión de Derechos Humanos del Estado de México (México).
} 


\begin{abstract}
In the current stage of Public International Law, access of the individual and organized civil society to international courts, just like participation in proceedings is a constant. In particular, through the institution of the amicus curiae, traditionally defined as the 'neutral bystander' who voluntarily intervenes in a dispute, assisting the court in the resolution of the case. This definition has evolved at the international level, so that in some cases the intervention of amici has been allowed to have an interest in the outcome of the procedures. However, the expression of interest is not expressly regarded as a criterion for determining the admission or rejection of amicus curiae in the rules of international criminal courts. In this sense, the Rome Statute of the International Criminal Court is no exception. For this reason, the present paper carries out a normative and jurisprudential analysis of the legal effects arising from the expression of interest in the pleadings submitted by civil society to the International Criminal Court.
\end{abstract}

Keywords: amicus curiae, civil society, interest, International Criminal Court.

\title{
1. Introducción
}

Actualmente, es posible afirmar que la institución del amicus curiae es el principal mecanismo de participación de los actores de la sociedad civil en la Corte Penal Internacional (CPI o 'Corte'). Además, las organizaciones no gubernamentales, grupos de juristas, instituciones académicas y expertos independientes, son el grupo más numeroso de cuantos solicitan participar como amici ante dicho órgano judicial ${ }^{1}$.

Esta circunstancia está en consonancia con el compromiso adquirido por la sociedad civil en relación a la promoción del Estatuto de Roma (ER) y el establecimiento de la 'Corte' a final de la década de los noventa del pasado siglo (Glasius, 2006). Dicho compromiso se ha reforzado a lo largo de los casi veinte años

\footnotetext{
${ }^{1} \mathrm{~A}$ los efectos de este trabajo, el vocablo amici designa a los terceros intervinientes en un procedimiento como amicus curiae. Es decir, se refiere exclusivamente a los sujetos que actúan como tal.
} 
que lleva en funcionamiento el citado tribunal; de modo que, como afirma Schiff (2008, 144), la sociedad civil, especialmente las organizaciones no gubernamentales, han pasado de ser cruciales en la creación de la Corte a vitales en el desarrollo de sus actividades.

La destacada participación de la sociedad civil en el ámbito de la justicia penal internacional, particularmente a través de la institución del amicus curiae, surge de la necesidad de dar voz a ciertos intereses y perspectivas que no suelen estar representados en los procedimientos penales internacionales (William et al, 2020).

En términos generales, podemos identificar dos tipos de intereses.

De un lado, la sociedad civil puede ejercer la representación directa de los intereses de las víctimas así identificadas y de los acusados, si, en virtud del tribunal o la etapa del procedimiento de que se trate, el amicus curiae es la única vía para que el órgano jurisdiccional conozca dichos intereses. Esta posibilidad, frecuentemente utilizada por organizaciones no gubernamentales en los inicios de la actividad judicial de la CPI, es residual e innecesaria en la actualidad, ya que la 'Corte', al igual que otros tribunales penales internacionales ${ }^{2}$, ha creado unidades especializadas en la representación de víctimas y acusados, como son la Oficina del Defensor Público para las Víctimas ('OPCV') y la Oficina del Defensor Público para el Acusado ('OPCD’). Además, los acusados sí son parte en los procedimientos, por lo que serán escasas las ocasiones en las que se precise la intervención de la sociedad civil para actuar como amicus curiae en representación directa de los intereses de víctimas y acusados.

Si bien, los amici pueden representar y dar voz a los intereses de las víctimas de forma abstracta y general (Kendall y Nouwen, 2013, 235), posibilidad que ha de desarrollarse con cautela, dadas las dificultades que puede tener la sociedad civil para identificar correctamente los intereses reales de las víctimas.

De otro lado, los actores de la sociedad civil pueden representar el interés público, entendiendo como tal el interés en proteger los bienes que pertenecen a la comunidad internacional en su conjunto (Benzing, 2006, 371). Así, con su intervención, los amici persiguen lograr la correcta administración de justicia. Es decir, se trata de que

\footnotetext{
${ }^{2}$ El Servicio de Apoyo al Acusado ('DSS') en las Salas Extraordinarias de la Corte de Camboya
} ('ECCC') responde a esta realidad. 
su opinión sobre la aplicación o interpretación de una norma, institución jurídica o cuestión de derecho específica, favorezca la idea de iustitia.

A la vista de lo anterior, podemos afirmar que la tradicional definición heredada del derecho anglosajón, que categorizaba al amicus curiae como un tercer interviniente neutral, se está desdibujando en la actualidad. Es más, hay quién considera que sería del todo irreal e innecesario esperar que los amici no representen un interés, ya que la mayoría sólo intervendrán si sirve a sus intereses de uno u otro modo (Wiik, 2016).

Como cabría esperar, esta postura no ha estado exenta de críticas, que principalmente se dirigen a rechazar la evolución del concepto tradicional de amicus curiae, poniendo en cuestión que la intervención de los amici en defensa de un interés particular contribuya a la obtención de la verdad en los procedimientos (Mavroidis, 2002). Además, también se ha señalado que los terceros que intervienen como amici estarían adquiriendo un derecho de participación que pone en riesgo los principios fundamentales clásicos del derecho procesal (Menétrey, 2010, 7).

En todo caso, es innegable la evolución de la institución del amicus curiae en el Derecho Internacional Penal, que, entre otras razones, obedece a la disparidad de criterios utilizados por los tribunales penales internacionales en el proceso de admisión de los escritos de amicus. En este sentido, nos planteamos si la expresión del interés es uno de estos criterios. La respuesta no está clara. Por una parte, los instrumentos jurídicos de tales órganos jurisdiccionales guardan silencio al respecto. Por otra, el análisis de la práctica judicial tampoco resulta esclarecedor, debido a las interpretaciones divergentes realizadas por los jueces sobre este particular.

Así, el Tribunal Internacional para la Antigua Yugoslavia, concluyó en el caso Miloševic que los amicus curiae no podían intervenir en representación de los intereses particulares del acusado al no ser partes en el proceso (Prosecutor v. Miloševic, 2001). En su opinión, la colaboración prestada por los amici durante el proceso, que sirvió para garantizar el respeto al principio del juicio justo ${ }^{3}$, no altera esta conclusión. Por su parte, en el caso Krajišvik, la Sala de Apelaciones del citado tribunal consideró que los jueces

\footnotetext{
${ }^{3}$ En este caso, la Sala de Primera Instancia asignó un amicus curiae al acusado con el propósito de que le asistiese en su defensa, ya que éste había solicitado defenderse a sí mismo. En opinión de la Sala, la asistencia del amicus curiae ayudaría a lograr el respeto al juicio justo y a garantizar los derechos del acusado.
} 
podían requerir la intervención de amici para que argumentasen a favor de una de las partes, si su actuación servía a los intereses de la justicia (Prosecutor v. Krajišvik, 2007).

El Tribunal Penal Internacional para Ruanda, en el caso Munyazaki, reconoció expresamente el carácter objetivo que debe reunir el interés de los terceros que intervienen en el procedimiento (Prosecutor v. Munyazaki, 2008). En relación al Tribunal Especial para Sierra Leona, cabe mencionar el caso Brima et al., en el que el citado órgano jurisdiccional fue contundente al señalar que la intervención de los amici debía responder al interés general (Prosecutor v. Brima et al, 2005).

En cuanto a la Corte Penal Internacional, todavía no hay suficientes trabajos académicos que estudien dicha institución, sobre todo en el ámbito de la literatura académica española y latinoamericana. A la vista de lo anterior, nos proponemos realizar un examen normativo y jurisprudencial de los efectos jurídicos derivados de la expresión de interés en los escritos de amicus presentados por la sociedad civil ante la Corte Penal Internacional.

Con este propósito, examinamos en un primer epígrafe los aspectos generales de la institución del amicus curiae en el ámbito de la justicia penal internacional. En el segundo epígrafe, estudiamos la interpretación del concepto de 'interés' en la normativa y jurisprudencia del citado tribunal. Para ello, nuestro método de análisis consiste en estudiar el marco jurídico aplicable a la institución del amicus curiae en el sistema de la 'Corte' y la interpretación del concepto de 'interés' en las decisiones adoptadas por los jueces del citado tribunal.

\section{Aspectos generales de la institución del amicus curiae en el ámbito de la justicia internacional penal}

\section{Definición, origen y evolución del concepto de amicus curiae}

Grosso modo, la institución del amicus curiae se podría definir como las presentaciones realizadas por un individual u organización que no es parte en un proceso, pero que ofrece voluntariamente su opinión al tribunal sobre una cuestión 
específica de carácter legal u otro aspecto relacionado, colaborando en la resolución del asunto (Gómez Orozco, 2016, 80).

En relación a su naturaleza jurídica, debemos advertir que se trata de un instituto procesal sujeto a la facultad discrecional de los tribunales internacionales, lo que supone que el órgano jurisdiccional invita o autoriza la intervención en el procedimiento de los amici sin concederles el estatus de partes procesales. Por ende, su interés difiere del que ostentan las partes en el litigio.

De esta manera, se hacen evidentes las disparidades existentes entre dicha institución y otras figuras jurídicas como la 'coadyuvancia', habitual ante el Tribunal de Justicia de la Unión Europea (artículo 40 del Estatuto del TJUE), y cuyo fin es la intervención de un tercero para respaldar los escritos de una de las partes. Para poder actuar como tal, es necesario que el tercer interviniente demuestre tener interés en el resultado del procedimiento.

Por cuanto se refiere a las funciones de la institución, Bartholomeusz (2005) identifica cuatro principales, a saber: proporcionar conocimientos jurídicos especializados al tribunal, especialmente en materias que se encuentren fuera de la competencia de los jueces; procurar información fáctica al tribunal; facilitar el acceso a la justicia a personas o entidades que no son parte en los procedimientos pero cuyos intereses podrían verse afectados por la decisión, e incluso, en cierta medida, representar el interés público. Por su parte, Williams, distingue tres funciones fundamentales que pueden ejercer los amicus curiae en los tribunales penales internacionales, incluyendo la Corte Penal Internacional. Así, junto a la tradicional función de información y consejo al tribunal, se reconocen las de representación de intereses particulares y la de comunicación de los intereses públicos (Williams et al, 2020, 19).

El nacimiento de la institución se sitúa en el derecho romano, aunque a partir del siglo IX se incorporó a la práctica judicial de Inglaterra y poco después a la mayoría de países de tradición jurídica anglosajona o 'common law', convirtiéndose en un instrumento habitual en la resolución de disputas de interés público en las que se presentaban posiciones muy controvertidas (McLauchlan, 2005, 266). 
Posteriormente, se produjo el 'trasplante' de la institución del amicus curiae a los sistemas jurídicos de tradición civilista (Kochevar, 2013). En especial, varios países latinoamericanos modificaron su legislación para acoger formalmente dicha institución en sus órganos judiciales. Este es el caso de la Corte Suprema de Justicia Argentina (CSJ, 2004), el Tribunal Constitucional del Perú (TC, 2004), y unos años más tarde, México (Código Federal de Procedimientos Civiles, 2011).

Paralelamente, su uso se 'popularizó' en los órganos internacionales de resolución de conflictos, siendo especialmente habitual en los mecanismos de protección regionales de los derechos humanos. En este sentido, resulta particularmente interesante la práctica de la Corte Europea de Derechos Humanos (CEDH) ${ }^{4}$.

Como señala Van del Eynde (2013), el concepto ha evolucionado desde su inclusión en el sistema jurídico del common law. De este modo, el amicus curiae se está convirtiendo en un medio para representar intereses particulares afectados en litigios en curso. Así, algunas definiciones de la institución que se refieren a la neutralidad y objetividad de los actores intervinientes estarían superadas. Por ejemplo, en su célebre diccionario de términos jurídicos, publicado por primera vez en 1926, Abbott (citado en Krislov, 1963) definió al amici como un espectador, que sin tener interés en la causa, realiza una observación sobre una cuestión de hecho o de derecho para informar al tribunal.

Unos años después, Krislov $(1963,704)$ afirmaba que en los Estados Unidos, país que ha usado frecuentemente esta institución, los escritos de amicus habían pasado de ser una fuente neutral de información a convertirse en un instrumento estratégico en manos de litigantes y terceras partes. En esta misma línea, Sands y Mackenzie subrayan que se trata de personas que tienen un fuerte interés en la materia (Sands y Mackenzie: 2009, 1).

\footnotetext{
${ }^{4}$ En la última década el número de escritos de amicus presentado por organizaciones no gubernamentales ha aumentado considerablemente. Entre 1998 y 2018, los casos en los que participaron estas organizaciones como amici ascendió a 68, respecto a las 12 participaciones registradas en el periodo comprendido entre 1989 y 1998 . Aunque en términos absolutos, los procedimientos en los que se presentaron y aceptaron escritos de amicus representan alrededor del 0.37 por ciento del número total de pronunciamientos. Este dato aproximativo se obtiene como resultado de dividir el número total de pronunciamientos emitidos por la CEDH desde 1959 (se tienen en cuenta sólo en los se ha decidido sobre el fondo de la cuestión 'decision on merits') para los escritos de amicus solicitados y aceptados hasta 2018 (ECHR Overview, 2019).
} 
Como hemos apuntado anteriormente, las diferencias interpretativas derivadas de la ausencia de regulación específica impiden conocer de antemano los criterios utilizados por las Salas para determinar la admisión o rechazo de las solicitudes de amicus curiae, incluyendo el sentido [admisorio o denegatorio] dado a las expresiones de interés en el resultado del procedimiento. A continuación, se realiza una revisión de los instrumentos normativos y la jurisprudencia de la 'Corte', con el fin de identificar los criterios formales y substantivos usados por las Salas para concluir la admisión de los amicus curiae.

Criterios formales y sustantivos de admisión de los amicus curiae en la Corte Penal Internacional

En el sistema de la CPI, el amicus curiae se articula a través de la Regla 103 de las Reglas de Procedimiento y Prueba, que en su inciso primero señala que:

La Sala, si lo considera conveniente para una determinación adecuada de la causa, podrá en cualquier etapa del procedimiento invitar o autorizar a un Estado, a una organización o a una persona a que presente, por escrito $u$ oralmente, observaciones acerca de cualquier cuestión que la Sala considere procedente.

Siguiendo este precepto cabe destacar que en relación a los criterios formales de admisión, tanto individuos como organizaciones podrán participar en los procedimientos como amicus curiae. Respecto a estas últimas, no se requiere que deban cumplir ningún requisito legal para intervenir como amicus curiae. Ni siquiera es necesario que las organizaciones estén inscritas o legalmente constituidas en un estado parte del ER, como sí ocurre en el sistema interamericano de derechos humanos respecto a la obligatoriedad de su constitución legal en un estado parte en la Comisión Americana de Derechos Humanos ${ }^{5}$. Por su parte, el artículo 36.2 del Convenio Europeo

\footnotetext{
${ }^{5}$ El sistema interamericano de derechos humanos tiene una estructura binaria, que comprende la Comisión Interamericana y la Corte Interamericana de Derechos Humanos (CorteIDH). Únicamente los estados parte de la 'Comisión' pueden llevar un caso ante la CorteIDH. En el artículo 44 de la Convención Americana de Derechos Humanos (CADH) se establecen las vías de participación de la sociedad civil ante la 'Comisión'. Dicho precepto permite que "cualquier persona o grupo de personas, o entidad no gubernamental legalmente reconocida en uno o más Estados miembros de la Organización,
} 
de Derechos Humanos, en el que se establece la institución del amicus curiae, alude al concepto de 'personas interesadas', sin especificar que debe considerarse como tal ${ }^{6}$.

Además, cualquier estado, sin necesidad de ser parte en el Estatuto de Roma, podrá solicitar su participación en los procedimientos como amicus curiae. De esta manera, la CPI está en sintonía con la mayoría de tribunales penales internacionales, que a excepción de las Salas Extraordinarias de las Cortes de Camboya ${ }^{7}$, permiten la participación a los estados. No obstante, por el momento, ninguno ha participado en la Corte Especial para Sierra Leona ni ante el Tribunal Especial para el Líbano, posiblemente debido a su limitada competencia territorial.

Por otra parte, la regla 103 RPP deja abierta la posibilidad de participar "en cualquier etapa del procedimiento". Si atendemos únicamente al tenor literal del citado precepto, aflora la duda de si cabría la participación en la fase previa al inicio del juicio oral. El problema surge al tratar de delimitar el alcance y significado del término 'procedimiento', al no haber disposiciones estatutarias que aclaren la cuestión. En consecuencia, debemos atender a la interpretación jurisprudencial del término.

Un análisis sobre este particular tuvo lugar a propósito del derecho de las víctimas a participar en el caso Lubanga. En aquella ocasión, la Sala de Primera Instancia I

pueda presentar a la Comisión peticiones que contengan denuncias o quejas de violación de esta Convención por un Estado parte".

${ }^{6}$ En la Corte Europea de Derechos Humanos (CEDH) coexisten desde 1998, año en el que entró en vigor el 'Protocolo número 11 al Convenio Para la Protección de los Derechos Humanos y de las Libertades Fundamentales, relativo a la reestructuración del mecanismo de control establecido por el Convenio, adoptado en Estrasburgo el 11 de mayo de 1999, entrada en vigor el 1 de noviembre de 1998, dos vías de participación de los actores de la sociedad civil en los procedimientos que tienen lugar ante la CEDH. La primera de ellas, establecida en el artículo 34 del citado 'Convenio Europeo' se refiere a la posibilidad de que individuos, organizaciones no gubernamentales, o grupo de individuales que se consideren víctimas de una violación por alguno de los estados parte, de los derechos reconocidos en dicho instrumento, presenten una demanda ante la CEDH. La segunda posibilidad, establecida en el artículo 36. 2 del Convenio Europeo y, que ya existía con anterioridad a la adopción del Protocolo 11, permite que las 'personas interesadas' puedan presentar observaciones por escrito o participar en la vista, por invitación del presidente del tribunal, si son de 'interés para la buena administración de justicia'. Es decir, la aceptación del escrito de amicus por parte del Presidente de la CEDH está condicionada a que éste ayude al tribunal en su tarea de resolver la disputa.

${ }^{7}$ La regla 33 de las 'Internal Rules' (12 de junio de 2007, revisadas el 16 de enero de 2015) Rev. 9, únicamente menciona expresamente a los individuos y organizaciones sin hacer referencia a los estados. En relación a la posibilidad de que individuos y organizaciones puedan presentar solicitudes de amicus curiae en el resto de tribunales internacionales, véase: rule 74 Rules of Procedure and Evidence of the International Criminal Tribunal for Yugoslavia, 14 de febrero de 1994, revisadas el 8 de julio de 2015, IT/32/Rev.50; rule 74 Rules of Procedure and Evidence of the International Criminal Tribunal for Ruanda, 29 de junio de 1995, ITR/3; rule 74 Rules of Procedure and Evidence of the Residual Special Court for Sierra Leone, entrada en vigor el 1 de enero de 2014; rule 131 Rules of Procedure and Evidence of the Special Tribunal for Lebanon, 20 de marzo de 2009, revisadas el 8 de febrero de 2012, STL/BD/2009/01/Rev.4. 
interpretó el término en sentido estricto para referirse a la fase de juicio oral (Prosecution vs. Lubanga, 2005). Sin embargo, actualmente, prevalece la interpretación extensiva del mismo, permitiéndose la participación de las víctimas desde la fase de examen preliminar (Prosecutor vs. Situation in Palestina, 2018). De manera similar, las Salas de la CPI han autorizado la participación de varios actores de la sociedad civil como amicus curiae en dicha fase.

En la situación de Bangladés/Myanmar (Prosecution vs. Situation in Bangladés/Myanmar, 2019), la Sala de Primera Instancia admitió algunos escritos de amicus presentados por organizaciones no gubernamentales, grupos de juristas e instituciones académicas a propósito de la cuestión sobre la competencia territorial, planteada por la Fiscalía en virtud del artículo 19.3 ER durante la fase de examen preliminar (Prosecution vs. Situation in Bangladés/Myanmar, 2018) ${ }^{8}$. En la situación en Afganistán, la Sala de Apelaciones ha autorizado a organizaciones locales de defensa de los derechos humanos y académicos la presentación de observaciones escritas y orales durante el examen en apelación del rechazo a la apertura de una investigación en el país (Prosecutor vs. Situation in Afghanistan, 2019), acordado por la Sala de Cuestiones Preliminares II el 12 de abril de $2019^{9}$.

Por otro lado, en cuanto al modo de iniciar el proceso de participación, la regla 103.1 RPP establece dos modalidades, por invitación o autorización de la Sala, que la praxis de la CPI ha canalizado a través de tres vías: la invitación concreta a un actor específico, generalmente expertos independientes en la materia; la apertura de un proceso abierto (las denominadas 'open calls') en el que suelen participar instituciones

\footnotetext{
${ }^{8}$ En la situación en Bangladés/Myanmar se investiga la deportación intencionada desde agosto de 2017 de 670.000 Rohingya, que vivían legalmente en Myanmar, a Bangladesh. La cuestión de competencia planteada por el Fiscal al amparo del artículo 19.3 ER examina si la Corte tiene competencia territorial para investigar los crímenes alegados, ya que los actos coercitivos relevantes en las deportaciones ocurrieron en Myanmar, un estado que no es parte en el Estatuto de Roma. El Fiscal alegó que el acto jurídico que constituye el crimen de deportación se cometió en Bangladés, que sí es estado parte del ER. El 14 de noviembre de 2019, la Sala de Cuestiones Preliminares III autorizó a la Fiscalía a que abriese una investigación en esta situación.

${ }^{9}$ El 12 de abril de 2019, la Sala de Cuestiones Preliminares II rechazó la solicitud presentada por la Fiscalía el 20 de noviembre de 2017 en la que ésta solicitaba autorización para abrir una investigación en Afganistán por la comisión de crímenes de lesa humanidad y de guerra en el país desde mayo de 2003. El argumento principal de la Sala de Cuestiones Preliminares II se basó en afirmar que la apertura de una investigación no redundaría en 'interés de la justicia', en el sentido que este concepto es comprendido en el artículo 53 ER. Tras examinar la apelación de la Fiscalía, el 5 de marzo de 2020 la Sala de Apelaciones desestimó la decisión de la Sala de Cuestiones Preliminares II autorizando la apertura de una investigación en Afganistán.
} 
académicas y grupos de juristas, y las solicitudes voluntarias enviadas por iniciativa de aquellos que desean intervenir como amicus curiae en los procedimientos, habitualmente organizaciones no gubernamentales.

La deficiente regulación de la institución del amicus curiae abarca los criterios sustantivos aplicables al examen sobre la admisibilidad de su participación en los procedimientos. En consecuencia, dichos criterios habrán de dilucidarse por las Salas de la CPI, lo que conlleva el consabido riesgo de que ejerzan un poder discrecional al decidir sobre la admisión de los escritos de amicus. En efecto, la regla 103 RPP establece un criterio general, indefinido y abstracto de admisión, condicionándose la misma a que la participación 'sea deseable para la determinación adecuada de la causa' [énfasis añadido]. Así, esta frase, que se reproduce con práctica literalidad en todos los tribunales penales internacionales ${ }^{10}$, da a los jueces un gran margen interpretativo y amplio poder de decisión.

En 2014, la Sala de Cuestiones Preliminares II identificó las tres consideraciones que las Salas acostumbran tienen en cuenta para dar significado al término 'deseable' (Prosecutor v. Ntaganda, 2014). La primera examina si el escrito de amicus versa sobre un asunto objeto de debate en el procedimiento en cuestión (Prosecutor v. Situation in Sudan, 2009). La segunda analiza si las observaciones realizadas pueden ayudar en la adecuada determinación del caso (Prosecutor v. Gbagbo, 2014; Prosecutor v. Ruto Sang, 2011; Prosecutor v. Lubanga, 2008). La tercera impone a la Sala la obligación de decidir sobre la admisión a la luz del deber de juzgar sin dilaciones indebidas (Prosecutor vs. Bemba, 2009).

En nuestra opinión, es necesario que las Salas realicen un mayor esfuerzo de concreción sobre los criterios utilizados para admitir o denegar los escritos de amicus. Respecto al tema que nos ocupa, el ER y las Reglas de Procedimiento y Prueba (RPP) guardan silencio sobre el régimen jurídico aplicable a la expresión del interés en el

\footnotetext{
${ }^{10}$ La frase se recoge de manera idéntica en la regla 74 de los estatutos del Tribunal Internacional Penal para la Antigua Yugoslavia, el Tribunal Internacional para Ruanda y la Corte Especial para Sierra Leona. En el caso de las Salas Extraordinarias de las Cortes de Camboya, la frase utilizada en la regla 33 es idéntica a la usada en los tribunales anteriormente citados pero incluye la palabra 'adjudicating'. Es decir, este tribunal introduce el requisito de que el amicus sea verdaderamente útil y sirva efectivamente a la resolución del caso. Por su parte, el Tribunal Especial para el Líbano, condiciona su admisión al hecho de que el amicus tenga la capacidad de asistir a la resolución del caso, no siendo suficiente con 'ser deseable' como es el caso de los tribunales citados al inicio de este párrafo.
} 
procedimiento por parte de los amici. De este modo, en lo que sigue trataremos de contribuir al análisis de esta cuestión.

\section{El concepto de interés en los amicus curiae presentados por la sociedad civil ante la Corte Penal Internacional}

Tal y como sucede en el resto de tribunales internacionales, cuyos estatutos no aclaran el término 'interés' en relación a los amicus curiae, el ER tampoco recoge definición alguna que nos sirva para delimitar su significado. Dicha ausencia dificulta saber en qué sentido es usado en la práctica judicial de la CPI. Por esta razón, en el apartado siguiente nos centramos en la interpretación del concepto de 'interés' en la Corte Penal Internacional. Nuestro propósito es examinar en qué medida la 'intervención interesada' de la sociedad civil tiene cabida en la 'Corte', y en su caso, qué clase de intereses son contemplados por las Salas.

El interés de la sociedad civil: ¿Requisito necesario o impedimento para la admisión del amicus curiae?

En el sistema de la Corte Penal Internacional no se obliga a los amici a revelar su interés en el caso, como sí sucede en otros tribunales penales internacionales ${ }^{11}$. En consecuencia, resulta difícil saber si la 'Corte' considera la expresión del interés como un requisito de admisión, o si por el contrario, siguiendo el concepto tradicional del amicus curiae deniega toda solicitud que incluya una declaración de interés en el procedimiento.

Por su parte, el análisis de la práctica judicial tampoco aclara esta cuestión. De un lado, el problema surge porque no todos los actores de la sociedad civil manifiestan tener un interés en sus escritos de amicus. Así, algunas organizaciones no gubernamentales como Human Rights Watch o la Women's Initiatives for Gender Justice incluyen en sus escritos una declaración de interés, aunque la gran mayoría no lo

\footnotetext{
11 Tribunal Especial para el Líbano. Practice Direction on Amicus Curiae submissions before the Special Tribunal for Lebanon, STL/PD/2012/05. 23 de febrero de 2012, artículo 3.d); Salas Extraordinarias de la Corte de Camboya. Information on Submission of Amicus Curiae Briefs to Supreme Court Chamber of the Extraordinary Chambers in the Court of Cambodia. 7 de marzo de 2011, artículo 2.a); Tribunal Internacional Penal para la Antigua Yugoslavia. Information Concerning the Submission of Amicus Curiae Briefs under Rule 74. IT/122. 27 de marzo de 1997, artículo 4.a).
} 
hacen (Williams et al., 2020, 149; Prosecutor v. Lubanga, 2006). A lo que hay que añadir que en ningún caso la 'Corte' ha denegado la intervención de un solicitante por este motivo. De esta manera, es imposible determinar el impacto positivo o negativo que tienen las declaraciones de interés en las decisiones de las Salas.

De otro lado, en el caso Al Bashir y la situación en Afganistán, la propia 'Corte' invitó a potenciales amici a indicar en su solicitud de participación su interés en intervenir como amicus curiae (Prosecutor v. Al Bashir, 2018, 3; Prosecutor v. Situation in Afganistán, 2019).

En suma, no se puede afirmar con rotundidad que en la 'Corte' caben, bajo toda circunstancia, las intervenciones 'interesadas', pero sí observamos cierta tendencia hacia la permisividad de los amicus curiae presentados por actores de la sociedad civil que expresan un interés, en consonancia con el desarrollo de dicha institución en el Derecho Internacional.

En segundo lugar, conviene preguntarse qué clase de intereses son bienvenidos en el sistema de la 'Corte'. Como es usual, nada se dice al respecto en los instrumentos jurídicos del citado órgano judicial. Respecto a esto, Williams et al. (2020) concluyen que los tribunales internacionales suelen interpretar el término 'interés' en un sentido más amplio que el meramente jurídico, tradicionalmente reservado a las partes procesales.

Esto nos obliga a distinguir entre los amici y las denominadas 'partes en el proceso' ante la Corte Penal Internacional, es decir: la Fiscalía y el acusado. Siguiendo esta lógica, la 'Corte' ha rechazado la participación de organizaciones no gubernamentales como amicus curiae en varias ocasiones por considerarla innecesaria, al haber realizado las partes y las víctimas las observaciones pertinentes durante el trascurso del procedimiento (Prosecutor v. Ruto et al., 2011, 8; Prosecutor v. Muthaura et al, 2011).

En ciertas ocasiones, los actores de la sociedad civil, especialmente las organizaciones no gubernamentales, dada la labor de acompañamiento y apoyo a las víctimas que llevan a cabo, participan como amicus curiae representando sus intereses ante la 'Corte'. No obstante, como ya hemos avanzado con anterioridad, esta posibilidad se reserva a las escasas situaciones en las que las víctimas no pueden prestar sus opiniones y observaciones por sí mismas, ni a través de sus representantes legales o la 
OPCV. En caso contrario, si los intereses de las víctimas están representados en el procedimiento de uno u otro modo, lo más probable es que la Sala rechace la solicitud como ya sucedió en el caso Ntaganda (Prosecutor vs. Ntaganda, 2014).

De esta manera, cabe concluir que el interés que siempre puede representar la sociedad civil es el público o colectivo. El propósito es dotar a los órganos judiciales internacionales de una nueva perspectiva sobre un tema en el que no tiene suficiente pericia. En este sentido, la Corte Penal Internacional no es una excepción. Por ello, al igual que la Corte Europea de Derechos Humanos (Mosley v. The United Kingdom, 2011), la 'Corte' condiciona la admisión de los escritos de amicus a la experiencia y conocimientos del amici sobre el asunto en cuestión.

\section{Conclusiones}

Debido a la ausencia de disposiciones normativas que desarrollen la institución del amicus curiae en la 'Corte', y ante la falta de jurisprudencia que aclare el sentido dado a las expresiones de interés formuladas por los amici, únicamente podemos hacer conjeturas al respecto. De cualquier manera, aunque no es posible afirmar con rotundidad que sea un requisito de admisión de los escritos de amicus, tampoco parece ser una causa para rechazarlos. Al menos cuando se trata de la representación de intereses públicos y colectivos por parte de actores de la sociedad civil.

A la vista de lo anterior, cabe apuntar dos ideas. La primera de ellas es que la definición del amicus curiae utilizada en la Corte Penal Internacional es similar a la empleada en otros tribunales penales internacionales. Así, este tribunal deja a un lado la tradicional definición del amicus curiae como un observador neutral, sirviéndose del interés que tienen quienes conocen en profundidad una materia para decidir respecto de un asunto sobre el que carece de conocimientos especializados. En segundo lugar, y en relación con lo anterior, hay que subrayar que en este caso el concepto de interés se asocia a la pericia, a la experiencia. En este sentido, la sociedad civil ejerce, como viene siendo habitual en el Derecho Internacional Público en general, y en el caso de la Corte Penal Internacional en particular, un rol de promoción y defensa del interés general y objetivo. 


\section{Bibliografía}

Benzing, M. (2006). Community interests in the procedure of international courts and tribunals. Law and Practice of International Courts and Tribunals, 5(3), 369-408.

Glasius, M. (2006). The ICC: A Global Civil Society Achievement. Routledge.

Gómez Orozco, J.A. (2016). Diccionario jurídico aplicado. Medellín: Librería Jurídica Sánchez R. Ldta.

Kendall, S., y Nouwen, S. (2013). Representational practices at the international criminal court: The gap between juridified and abstract victimhood. Law and Contemporary Problems, 76 (3\&4), 235-262.

Kochevar, S. (2013). 'Amici curiae in civil law jurisdictions'. Yale Law Journal, 122(6), 1653-1669.

Krislov, S. (1963). 'The Amicus Curiae Brief: From Friendship to Advocacy', The Yale Law Journal, 72, 694-721.

McLauchlan, J. S. (2005). Congressional participation as amicus curiae before the U.S. Supreme Court. New York: LFB Scholarly Pub.

Menétrey, S. (2010). L’amicus curiae, vers un principe commun de droit procédural?. Dollaz.

Pascual Vives, F.J. (2011). El desarrollo de la institución del amicus curiae en la jurisprudencia internacional. Revista Electrónica de Estudios Internacionales, 21, 1-37.

Sands, P. J y Mackenzie, R. (2009). International Courts and Tribunals, Amicus Curiæ. In Wolfrum, R. (ed.), The Max Planck Encyclopedia of Public International Law. Oxford University Press.

Schiff, B. (2008). Building the International Criminal Court. New York: CUP.

Van den Eynde, L. (2013). An Empirical look at the amicus curiae practice before the European Court of Human Rights. Netherlands Quaterly of Human Rights, 31, 271-313. 
Wiik, A (2016). Amicus Curiae before International Courts and Tribunals. Hart Publishing.

Williams, S, Woodaver, H, Palmer, E. (2020). The Amicus Curiae in International Criminal Justice. Hart Publishing.

\section{Instrumentos jurídicos internacionales}

Consejo de Europa (CdE). Convención Europea de Derechos Humanos. Roma, 4 de noviembre de 1950.

Corte Penal Internacional (CPI). Estatuto de Roma. Roma, 17 de julio de 1998.

Organización de Estados Americanos (OEA). Convención Americana de Derechos Humanos (Pacto de San José), San José, Costa Rica. 7 al 22 de noviembre de 1969.

\section{Jurisprudencia}

CPI, Sala de Apelaciones. (2019). Prosecutor vs. Situation in Afghanistan. Decision on the participation of amici curiae, the Office of Public Counsel for the Defence and the cross-border victims. 24 de octubre (ICC-02/17 OA OA2 OA3 OA4).

CPI, Sala de Cuestiones Preliminares. (2018). Prosecutor v. Situation in Bangladesh. Decision on the Request of Mr. Mohammed Hadi Zakerhossein for Leave to Submit Amicus Curiae Observations. 7 de junio (ICC-RoC46 (3)-01/18-12).

CPI, Sala de Cuestiones Preliminares II. (2014). Prosecutor v. Bosco Ntaganda. Decision on the application by the Redress Trust to submit Amicus Curiae observations. 18 de febrero (ICC-01/04-02-06-259).

CPI, Sala de Cuestiones Preliminares I. (2014). Prosecutor v. Laurent Gbagbo. Decision on a Request for leave to submit Amicus Curiae observations. 4 de junio (ICC02/11-01/11-653).

CPI, Sala de Apelaciones. (2008). Prosecutor v Thomas Lubanga Dyilo. Decision on "Motion for Leave to File Proposed Amicus Curiae Submission of the International Criminal Bar Pursuant to Rule 103 of the Rules of Procedure and Evidence”. 22 de abril (ICC-01/04-01/06-1289). 
CPI, Sala de Apelaciones. (2009). Prosecutor v. Jean Pierre Bemba Gombo. Decision on the application of 14 september 2009 for participation as an Amicus Curiae. 9 de noviembre (ICC-01/05-01/08-602).

CPI, Sala de Cuestiones Preliminares I. (2009). Prosecutor v. Situation in Darfur, Sudan. Decision on Application under Rule 103. 4 de febrero (ICC-02/05-185).

CPI, Sala de Cuestiones Preliminares I. (2018). Prosecutor v. Ruto. Decision on the 'Request for leave to submit Amicus Curiae observations by the Inter Commission of Jurits (pursuant to rule 103 of the Rules). 29 de mayo (ICC-RoC46(3)-01/18).

CPI, Sala de Cuestiones Preliminares II. (2011). Prosecutor v. William Samoei Ruto, Henry Kiprao Kosgey and Joshua Arap Sang. Decision on the 'Request for leave to submit Amicus Curiae observations pursuant to Rule 103 of the Rules of Procedure and Evidence. 13 de junio (ICC-01/09-01/11-124).

CPI, Sala de Cuestiones Preliminares II. (2011). Prosecutor v. Francis Kirimi Muthaura, Uhuru Muigui Kenyatta and Mohammed Hussein Ali. Decision on the 'Request by Ms. Moraa Gesicho to Appear as Amici Curiae. 12 abril (ICC-01/09$02 / 11)$.

CPI, Sala de Primera Instancia VI. (2014). Prosecutor v. Bosco Ntaganda. Decision on the request by the Redress Trust and Avocats sans Frontières for leave to submit amicus curiae observations. 24 de septiembre (ICC-01/04-02/06-377).

CPI, Sala de Cuestiones Preliminares I. (2006). Prosecutor v. Thomas Lubanga Dylo. Request submitted pursuant to rule 103 (1) of the rules of procedure and evidence for leave to participate as Amicus Curiae in the article 61 confirmation proceedings (with confidential annex 2). 7 de septiembre (ICC-01/04-01/06-403).

CPI, Sala de Apelaciones. (2018). Prosecutor v. Oman Hassan Ahmad Al Bashir. Order inviting expressions of interest as amici curiae in judicial proceedings (pursuant to rule 103 of the Rules of Procedure and Evidence). 29 de marzo (ICC-02/05-01/09-330).

CEDH (Corte Europea de Derechos Humanos). Mosley v. The United Kingdom. No. 48009/08. 10 de mayo de 2011. 
CESL (Corte Especial para Sierra Leona). Prosecutor v. Alex Tamba Brima, Ibrahim Bazzy Kamara y Santigie Borbor Kanu, Caso n SCSL-04-15-T, 20 de mayo de 2005.

TPIY (Tribunal Penal Internacional para la Antigua Yugoslavia). The Prosecutor $v$. Slobodan Milosevic, "Order Inviting Designation of Amicus Curiae", Case No. IT-9937-PT, 31 de agosto de 2001.

TPIY. The Prosecutor v. Momčilo Krajišvik, "Decision on Momčilo Krajišvik's Request to Self-Represent, on Counsel's Motions in relation to appointment of amicus curiae, and the Prosecution Motion of 16 february 2007", Case No. IT-00-39-A, 11 de mayo de 2007.

TPIR (Tribunal Penal Internacional para Ruanda). The Prosecutor v. Yussuf, "Decision on Request from the Republic of Rwande for Permission to File an Amicus Curiae Brief", Case No. ICTR-97-36-RIlbis, 18 de julio de 2008.

\section{Otros documentos}

Código Federal de Procedimientos Civiles (2011). Enmendado el 30 de agosto de 2011. Diario Oficial de la Federación (DO). 24 de febrero de 1943 (Mex).

Corte Suprema de Justicia de Argentina (2004). Acordada No. 28/2004-CSJ, 20 de julio de 2004. [CXII-30. 4 55] B.O. 6 (Arg).

Corte Europea de Derechos Humanos. Overview 1959-2019. https://www.echr.coe.int/Documents/Overview_19592019_ENG.pdf

Salas Extraordinarias de la Corte de Camboya. Information on Submission of Amicus Curiae Briefs to Supreme Court Chamber of the Extraordinary Chambers in the Court of Cambodia. 7 de marzo de 2011.

Tribunal Constitucional del Perú. (2004). Resolución Administrativa No. 095-2004P/TC, Sept. 14, 2004, Art. 13-A (Perú).

Tribunal Especial para el Líbano. Practice Direction on Amicus Curiae submissions before the Special Tribunal for Lebanon, STL/PD/2012/05. 23 de febrero de 2012.

Tribunal Internacional Penal para la Antigua Yugoslavia. Information Concerning the Submission of Amicus Curiae Briefs under Rule 74. IT/122. 27 de marzo de 1997. 